\title{
CNS-Derived Interleukin-4 Is Essential for the Regulation of Autoimmune Inflammation and Induces a State of Alternative Activation in Microglial Cells
}

\author{
Eugene D. Ponomarev, ${ }^{\star}$ Katarzyna Maresz, ${ }^{*}$ Yanping Tan, and Bonnie N. Dittel \\ BloodCenter of Wisconsin, Blood Research Institute, Milwaukee, Wisconsin 53201-2178
}

\begin{abstract}
Regulation of inflammation in the CNS is essential to prevent irreversible cellular damage that can occur in neurodegenerative diseases such as multiple sclerosis (MS). We investigated the role of interleukin-4 (IL-4) in regulating CNS inflammation using the animal model of MS, experimental autoimmune encephalomyelitis (EAE). We found that CNS-derived IL-4 was a critical regulator because mice with a deficiency in IL-4 production in the CNS, but not the periphery, had exacerbated EAE associated with a significant increase in the absolute number of infiltrating inflammatory cells. We also found that CNS-resident microglial cells in both the resting and activated state produced the protein Ym1, which is a marker of alternatively activated macrophages (aaM $\Phi$ s), in an IL-4-dependent manner. This aaM $\Phi$ phenotype extended to the lack of nitric oxide (NO) production by activated microglial cells, which is a marker of classically activated macrophages. We also show that IL-4 induced the expression of Ym1 in peripheral infiltrating macrophages, which also produce NO. Thus, macrophages that migrate into the CNS exhibit a dual phenotype. These data indicate that IL-4 production in the CNS is essential for controlling autoimmune inflammation by inducing a microglial cell aaM $\Phi$ phenotype. Macrophages that have undergone alternative activation have been shown to be important in tissue repair; thus, our results suggest a new role for microglial cells in the regulation of inflammation in the CNS.
\end{abstract}

Key words: activation; autoimmunity; encephalomyelitis; interleukin; macrophage; microglia; multiple sclerosis; neuroinflammation

\section{Introduction}

Microglial cells are myeloid lineage cells resident to the CNS. In the normal CNS, microglial cells have been shown to survey their surrounding microenvironment (Nimmerjahn et al., 2005). However, in a pathological state, microglial cells have the capacity to become rapidly activated, changing both their morphology and phenotype (Nelson et al., 2002). It is becoming increasingly clear that microglial cells have multiple functions in the CNS, including antigen presentation, phagocytosis, and cytokine production, which, depending on the disease state, can be pathogenic or protective. In multiple sclerosis (MS), microglial cells are thought to perform all of these functions (Carson, 2002). Although much is known about microglial cell activation, little is known about how they serve a protective role in the CNS.

Interleukin-4 (IL-4) is a well described immune regulatory cytokine able to suppress inflammation. Its role in regulating inflammation within the CNS has been demonstrated in the mouse model of MS, experimental autoimmune encephalomy-

Received April 27, 2007; revised Aug. 12, 2007; accepted Aug. 20, 2007.

This work was supported by National Institutes of Health Grant R01 NS46662-01A1 and the BloodCenter Research Foundation. We thank Dr. Shioko Kimura for the rabbit polyclonal anti-Ym1/2 antibody, Shelley Morris-Islo for assistance with the mice, and Drs. Peter Newman and Reema Goel for assistance with the NO analysis.

*E.D.P. and K.M. contributed equally to this work.

Correspondence should be addressed to Dr. Bonnie N. Dittel, P.0. Box 2178, Milwaukee, WI 53201-2178. E-mail: bonnie.dittel@bcw.edu.

DOI:10.1523/JNEUROSCI.1922-07.2007

Copyright $\odot 2007$ Society for Neuroscience $\quad$ 0270-6474/07/2710714-08\$15.00/0 elitis (EAE), whereby mice deficient in IL-4 exhibited more severe EAE clinical disease (Bettelli et al., 1998; Falcone et al., 1998). An importance for IL-4 within the CNS was demonstrated when IL-4 was delivered to the CNS by IL-4 retroviral-transduced T-cells or by a nonreplicative herpes simplex type 1 viral vector, resulting in the amelioration of EAE (Shaw et al., 1997; Furlan et al., 1998).

One mechanism for how IL-4 is thought to regulate inflammation is by influencing the phenotype and function of macrophages. The activation of macrophages in the presence of IL-4 results in an alternatively activated ( $\mathrm{a} M \Phi)$ phenotype first characterized by an increase in the mannose receptor and major histocompatibility complex (MHC) class II expression (Stein et al., 1992). Recently, the chitinase family protein $\mathrm{Ym} 1$ was shown to be an excellent marker of aaM $\Phi$ s (Chang et al., 2001; Raes et al., 2002). The primary function of aaM $\Phi$ s is thought to be the resolution of inflammation and promotion of wound healing. Both events are important in the CNS for the recovery from MS and EAE, which requires resolution of the immune inflammatory infiltrate and remyelination of neurons. Macrophages activated classically (caM $\Phi$ s) by interferon- $\gamma($ IFN- $\gamma$ ) produce nitric oxide $(\mathrm{NO})$, are proinflammatory, and drive chronic inflammation and tissue injury, events involved with MS and EAE pathogenesis.

In this study, we examined the macrophage phenotype that the CNS microenvironment supports under normal conditions and during EAE pathological disease. We found that microglial cells produced both IL-4 and Ym1, with both increasing after activation during EAE. CNS-infiltrating macrophages expressed 
similar levels of Ym1 protein. One major difference between the two myeloid populations was the expression of $\mathrm{NO}$, which was expressed by the infiltrating macrophages but not by microglial cells. Using mice deficient in IL-4 production or signaling only within the CNS, we found that the aaM $\Phi$ phenotype was IL-4 dependent. In these same mice, EAE clinical disease was more severe. These data suggest that the CNS microenvironment, by the production of IL-4, actively regulates the function of macrophages by promoting an aa $\Phi \Phi$ phenotype that is required for the control of CNS inflammation.

\section{Materials and Methods}

Mice. B10.PL $\left(\mathrm{H}-2^{\mathrm{u}}\right)$ and $(\mathrm{B} 10 . \mathrm{PL} / \mathrm{C} 57 \mathrm{BL} / 6) \mathrm{F}_{1}\left(\mathrm{H}-2^{\mathrm{uxb}}\right)$ mice were bred locally. C57BL/6J, BALB/cJ, C57BL/6-Ill ${ }^{\mathrm{tm} 1 \mathrm{Nnt} / J}\left(\mathrm{IL}-4^{-1-}\right)$ and BALB/

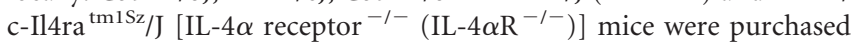
from The Jackson Laboratory (Bar Harbor, ME). The MBP-TCR transgenic mice have been described previously (Dittel et al., 1999). All animal protocols were approved by the Medical College of Wisconsin Institutional Animal Care and Use Committee.

Peptides and antibodies. The MBP Ac $\mathrm{A}_{1-11}$ and $\mathrm{MOG}_{35-55}$ peptides were synthesized by the BloodCenter of Wisconsin, Blood Research Institute Peptide Core. Anti-mouse H-2K ${ }^{\mathrm{b}}$-FITC was purchased from PharMingen (San Diego, CA). Anti-mouse H-2D ${ }^{\mathrm{d}}$-FITC, CD11b-phycoerythrin (PE)-Cy5 H-2D ${ }^{\mathrm{b}}$-biotin, and CD45-allophycocyanin (APC)-Cy5 monoclonal antibody $(\mathrm{mAb})$ were purchased from Biolegend (San Diego, CA). Streptavidin (SA)-PE, SA-APC-Cy7, and CD45-PE were purchased from eBioscience (San Diego, CA).

Irradiation bone marrow chimeras. Bone marrow (BM) chimeras were generated as described previously (Ponomarev et al., 2005b), by transplanting $\mathrm{H}-2^{\text {uxb }}$ donor $\mathrm{BM}$ cells from (B10.PL/C57BL/6) $\mathrm{F}_{1}$ mice into B10.PL (H-2 $\left.{ }^{\mathrm{u}}\right), \mathrm{C} 57 \mathrm{BL} / 6\left(\mathrm{H}-2^{\mathrm{b}}\right)$, or C57BL/6-IL-4 $4^{-1-}\left(\mathrm{H}-2^{\mathrm{b}}\right)$ recipient mice. Donor cells were differentiated from recipient B10.PL or C57BL/6 mice by the expression of $\mathrm{H}-2 \mathrm{~K}^{\mathrm{b}}$ or $\mathrm{H}-2 \mathrm{D}^{\mathrm{d}}$, respectively. In these chimeras, $97 \%$ of the CNS-resident cells are $\mathrm{CD} 11 \mathrm{~b}^{+} \mathrm{CD} 45^{\text {low }}$ microglial cells that are recipient derived as indicated by their lack of $\mathrm{H}-2 \mathrm{~K}^{\mathrm{b}}$ expression. The remaining $3 \%$ are $\mathrm{H}-2 \mathrm{~K}^{\mathrm{b}+}$ donor-derived perivascular macrophages. In the periphery, $\mathrm{H}-2 \mathrm{~K}^{\mathrm{b}}$ was shown to be expressed by $98 \%$ of peritoneal macrophages and $91 \%$ of $\mathrm{CD} 11 \mathrm{~b}^{+}$peripheral blood mononuclear cells. In the generation of the chimeras, the lethal irradiation does not result in activation of microglial cells after a 8-10 week engraftment period (Ponomarev et al., 2005b).

EAE induction. In B10.PL chimera mice, EAE was induced by the adoptive transfer of MBP-specific encephalitogenic T-cells generated as described previously (Dittel et al., 1999). Briefly, mice are sublethally irradiated $(360 \mathrm{rad})$ before adoptive transfer of $0.5-1 \times 10^{6}$ encephalitogenic T-cells. We found that the low-dose irradiation does not result in microglial cell activation (Ponomarev et al., 2005b). In C57BL/6-IL-4 ${ }^{-1-}$ chimera mice, EAE was induced by immunization with $400 \mu \mathrm{g}$ of $\mathrm{MOG}_{35-55}$ peptide in complete Freund's adjuvant (Chondrex, Redmond, WA). Pertussis toxin (200 ng) (List Biological Labs, Campbell, CA) was injected intraperitoneally at the time of immunization and $48 \mathrm{~h}$ later. Individual animals were scored using the following scale: (0) no disease, (1) limp tail and/or hindlimb ataxia, (2) hindlimb paresis, (3) hindlimb paralysis, (4) hindlimb and forelimb paralysis, and (5) death.

Flow cytometry and cell sorting. Total mononuclear cells were isolated and pooled from the brains and spinal cords of three to five unmanipulated or chimeric mice with EAE on the day of peak of disease (day 10 for B10.PL chimeras and day 14 for C57BL/6 and C57BL/6-IL-4 ${ }^{-1-}$ chimeras) as described previously (Ponomarev and Dittel, 2005). Analysis of CNS mononuclear cells isolated from chimeric animals with EAE was performed using CD11b-PE-Cy5, CD45-PE-Cy7, and H-2K ${ }^{\mathrm{b}}$-FITC (B10.PL) or H-2K ${ }^{\mathrm{d}}$-FITC (C57BL/6) (Ponomarev et al., 2005b). In the normal CNS, perivascular macrophages were distinguished from microglial cells by forward and size scatter parameters (Ponomarev et al., 2005b). For cell sorting, CNS mononuclear cells were stained for expression of $\mathrm{H}-2 \mathrm{~K}^{\mathrm{b}}, \mathrm{CD} 45$, and $\mathrm{CD} 1 \mathrm{lb}$ and then sorted into four populations: (1) peripheral infiltrating macrophages $\left(\mathrm{CD} 11 \mathrm{~b}^{+}, \mathrm{CD} 45^{\text {high }}\right.$, and $\left.\mathrm{K}^{\mathrm{b}+}\right)$,
(2) resting microglial cells $\left(\mathrm{CD} 11 \mathrm{~b}^{+}\right.$and $\left.\mathrm{CD} 45^{\text {low }}\right),(3)$ activated microglial cells $\left(\mathrm{CD}_{11 \mathrm{~b}}{ }^{+}\right.$and $\left.\mathrm{CD} 45^{\text {high }}\right)$, and (4) lymphocytes $\mathrm{CD} 11 \mathrm{~b}^{-} \mathrm{CD} 45^{\text {high }}$ (Ponomarev et al., 2005b). For the detection of cytoplasmic Ym1, we used the Cytofix/Cytoperm kit for intracellular cytokine staining from BD Biosciences (San Diego, CA). For permeabilization, the cells were stained for the expression of surface markers and then were centrifuged and resuspended in Cytofix/Cytoperm solution and incubated for $20 \mathrm{~min}$ on ice. The cells were then washed in Perm/Wash solution and blocked with $10 \%$ goat serum (Zymed, San Francisco, CA) and $20 \%$ FBS in Perm/Wash solution for $30 \mathrm{~min}$ at room temperature. After blocking, the cells were washed again with Perm/Wash solution and resuspended in Perm/Wash solution containing rabbit polyclonal anti$\mathrm{Ym} 1 \mathrm{Ab}$, which recognizes both $\mathrm{Ym} 1$ and $\mathrm{Ym} 2$ proteins (Chang et al., 2001) or rabbit serum (Sigma-Aldrich, St. Louis, MO). After a $1.5 \mathrm{~h}$ incubation at room temperature, the cells were washed with Perm/Wash solution and then incubated in Perm/Wash solution containing antirabbit IgG-Biotin (Jackson ImmunoResearch, West Grove, PA) for 20 $\mathrm{min}$ at room temperature. The cells were then washed with Perm/Wash solution and incubated in Perm/Wash solution containing SA-PE (Caltag, Burlingame, CA) for $15 \mathrm{~min}$ at room temperature. Finally, the cells were washed two times with $1 \mathrm{ml}$ of Perm/Wash solution, fixed in $1 \%$ paraformaldehyde, and analyzed on LSR II Flow Cytometer (BD Biosciences).

Detection of NO. The production of NO in live cells was determined using a 4-amino-5-methylamino-2', $7^{\prime}$-difluorofluorescein (DAF-FM) diacetate probe (Invitrogen, Carlsbad, CA). Briefly, $\sim 0.5 \times 10^{6} \mathrm{CNS}$ mononuclear cells were incubated with $10 \mu \mathrm{M}$ DAF-FM diacetate and the anti-Fc receptor $\mathrm{mAb} 2.4 \mathrm{G} 2$ for $30 \mathrm{~min}$, before cell surface staining.

Real-time PCR. Total mRNA was isolated using the Dynabeads mRNA Direct kit (Dynal Biotech ASA, Oslo, Norway). cDNA was synthesized, Ym1 and IL-4 were quantitated by real-time reverse transcription (RT)PCR using SYBR-Green as the detection agent, and threshold values of Ym1 and IL-4 were normalized to glyceraldehyde-3-phosphate dehydrogenase (GAPDH) expression using standard curves as described previously (Ponomarev et al., 2004). The following sequence-specific primers were used in real-time PCR: GAPDH primers have been described previously (Ponomarev et al., 2004); Ym1 (Raes et al., 2002), which amplifies both Ym1 and Ym2, forward, 5'-GGGCATACCTTTATCCTGAG-3'; reverse, 5'-CCACTGAAGTCATCCATGTC-3'; FIZZ1 (Raes et al., 2002), forward, 5'-TCCCAGTGAATACTGATGAGA-3'; reverse, 5'-CCACTCTGGATCTCCCAAGA-3'; and IL-4 (Overbergh et al., 1999), forward, 5'-ACAGGAGAAGGGACGCCAT-3'; reverse, 5'-GAAGCCCTACAGACGAGCTCA-3'. Specificity of the RT-PCR was controlled by the generation of melting curves, PCR efficiencies were $100 \pm 15 \%$, and correlation coefficients were $0.97-0.99$.

Thioglycollate macrophage isolation and microglial cell culture. Peritoneal macrophages were obtained using thioglycollate as described previously (Raes et al., 2002) and incubated in DMEM media (Cellgro; Mediatech, Herndon, VA) with $10 \%$ FBS supplemented with IL-4 (200 U/ml; Genzyme, Cambridge, MA) or IFN- $\gamma(150 \mathrm{U} / \mathrm{ml}$; eBioscience) combined with lipopolysaccharide (LPS; $100 \mathrm{ng} / \mathrm{ml}$; Sigma-Aldrich) for $24 \mathrm{~h}$ before staining for Ym1. Microglial cell lines were generated from CNS mononuclear cells isolated from 4-week-old B10.PL as described previously (Ponomarev et al., 2005a). After 4 weeks, the microglial cell cultures were stimulated in the same manner as peritoneal macrophages.

\section{Results \\ Microglial cells express high levels of mRNA for IL-4 and Ym1 in the CNS}

It was previously demonstrated that IL-4-deficient mice are more susceptible to EAE, but the mechanism of this phenomenon is still unclear (Bettelli et al., 1998; Falcone et al., 1998). One possible mechanism of how IL-4 inhibits EAE is the induction of aaMФs, which have a low capacity to support proliferation of autoimmune Th1 cells (Edwards et al., 2006). In this study, we hypothesized that IL-4 production by CNS-resident cells induced an aaM $\Phi$ phenotype in microglial cells. To test this hypothesis, we first analyzed the expression of IL- 4 mRNA in the spinal cord of unmanipulated mice, and using real-time RT-PCR, we did not 
detect message for IL-4 in the total spinal cord homogenate (data not shown). In contrast, IL-4 message was detected in purified CNS mononuclear cells, of which $\sim 95 \%$ are microglial cells (Fig. 1A) (Ponomarev et al., 2005b). The inability to detect mRNA for IL-4 in total spinal cord homogenate is likely caused by the low level of expression by microglial cells, which constitute $<10 \%$ of the CNS cellularity, being below the sensitivity threshold of real-time RT PCR.

Because IL-4 is known to induce the expression of Ym1 (Webb et al., 2001), we examined its expression in microglial cells and found that it was expressed at a high level (Fig. 1B). To investigate whether microglial cells upregulate IL-4 and Ym1 during EAE, we used MHC class I mismatched BM chimeras to discriminate CNS-resident microglial cells from blood-derived peripheral macrophages (Ponomarev et al., 2005b). In these chimeras, the peripheral infiltrating macrophages are distinguished from microglial cells by the expression of $\mathrm{H}-2 \mathrm{~K}^{\mathrm{b}}$. Microglial cells that become activated during EAE are characterized by a CD $45^{\text {high }}$ phenotype, when compared with resting microglial cells, which are CD45 ${ }^{\text {low }}$ (Sedgwick et al., 1991; Ponomarev et al., $2005 b)$. At the peak of the EAE disease course, we sorted populations of resting CD $45^{\text {low }}$ microglial cells, activated CD $45^{\text {high }}$ microglial cells, and peripheral infiltrating macrophages and analyzed the level of mRNA expression for IL- 4 and Ym1. The level of IL-4 expression was increased by approximately fourfold in both microglial cell populations compared with mice without EAE (Fig. 1A). In contrast, peripheral infiltrating macrophages expressed IL- 4 mRNA at a level $\sim 100$ lower than microglial cells (Fig. 1A). Unlike IL-4, the expression of Ym1 was not altered in the $\mathrm{CD} 45^{\text {low }}$ microglial cells during EAE. However, after activation $\left(\mathrm{CD} 45^{\text {high }}\right)$, the Ym1 message level was increased by fivefold (Fig. $1 B$ ). As with IL-4, the peripheral infiltrating macrophages expressed Ym1 at an $\sim 80$-fold lower level than the microglial cells (Fig. $1 \mathrm{~B}$ ). These data indicate that CNS microglial cells exhibit properties of aaM $\Phi$ s, as indicated by high levels of mRNA expression for both IL-4 and Ym1.

In this study, the Yml primers used amplify both Yml and $\mathrm{Ym} 2$, but because $\mathrm{Ym} 2$ is primarily expressed in the stomach, we are likely detecting only Ym1 (Jin et al., 1998; Nio et al., 2004). We confirmed this by using PCR primers specific for Ym1 or Ym2, as described previously (Welch et al., 2002), and only detected message for Ym1 in the CNS (data not shown).

To confirm that Ym1 was detecting an aaM $\Phi$, we also examined the expression of an additional marker of these cells, FIZZ1 (Raes et al., 2002). We found that FIZZ1 was highly expressed by microglial cells in both normal and EAE mice and that this level increased over 10 -fold after activation during EAE (Fig. $1 C$ ). The infiltrating macrophages expressed FIZZ1 message at a level similar to activated microglial cells. These cumulative data demonstrate that the CNS microenvironment supports an aaM $\Phi$ phenotype.

\section{Resting microglial cells express $\mathrm{Ym} 1$ protein in the absence of NO production}

Because we found that microglial cells in vivo expressed mRNA for Ym1 (Fig. 1B), we next investigated whether they express Ym1 protein. To quantitate the level of Ym1 expression by flow cytometry, we developed an intracellular staining method. We first validated our technique using peritoneal macrophages activated classically with IFN- $\gamma /$ LPS or alternatively with IL-4. As expected, $57 \%$ of aaM $\Phi$ s were positive for $\mathrm{Ym} 1$, whereas caM $\Phi_{\mathrm{s}}$ were negative for Ym1 protein expression (Fig. 2A). We then examined the level of Ym1 protein expression by CNS-resident microglial cells versus blood-derived CNS perivascular macrophages and found that $25 \%$ of microglial cells expressed Ym1, whereas only $9 \%$ of perivascular macrophages were positive (Fig. $2 B)$. Over three experiments, this difference was statistically significant (Fig. 2D).

Another feature that distinguishes caM $\Phi$ s from aaMФs is the ability of caMФs to produce NO (Edwards et al., 2006). We compared NO production by microglial cells and perivascular macrophages in the normal CNS by flow cytometry using a DAF diacetate probe that can penetrate the cell and becomes fluorescent after binding to NO. We found that the mean fluorescence intensity (MFI) of the fluorescent probe was twofold to threefold higher in perivascular macrophages than in microglial cells (Fig. $2 C, E)$. This difference was statistically significant over three experiments $(p<0.01)$ (Fig. $2 E)$. These data further suggest that a subpopulation of microglial cells exhibits an aaM $\Phi$ phenotype in the normal CNS, characterized by a high level of Ym1 expression in the absence of NO production.

\section{During EAE, activated microglial cells upregulate Ym1 protein expression in the absence of NO production}

Studies have suggested that microglial cells express inducible nitric oxide synthase (iNOS) in the CNS during EAE (Okuda et al., 1995; Tran et al., 1997); however, these studies did not differentiate between microglial cells and infiltrating macrophages. Thus, we quantitatively analyzed the level of $\mathrm{NO}$ production and $\mathrm{Ym} 1$ protein expression by the three microglial cell populations and peripheral infiltrating macrophages. Because Ym1 was reported to be predominantly expressed by myeloid cells, we used the CNS lymphocyte population as a negative control for $\mathrm{Ym} 1$ expression (Fig. 3A). During EAE, the percentage of $\mathrm{Ym} 1{ }^{+} \mathrm{CD} 45^{\text {low }}$ microglial cells increased to $89 \%$, which was further increased to $99 \%$ in the CD $45^{\text {high }}$ activated microglial cell population (Fig. $3 A$ ). Thus, during EAE, the majority of microglial cells expressed an alternatively activated phenotype. This is likely caused by increased production of IL-4 by the microglial cells (Fig. 1). The high level of Ym1 protein expression by microglial cells is consistent with their high mRNA expression during EAE (Fig. $1 A$ ). We also found that during EAE, peripheral infiltrating macrophages expressed a high level of Ym1 protein (Fig. 3A). This result was unexpected, because peripheral infiltrating macrophages expressed a low level of mRNA for Ym1 during EAE (Fig. $1 A$ ). These data indicate that Ym1 mRNA and protein levels are not always correlative. The 


\section{A IFN- $\gamma /$ LPS}

IL-4
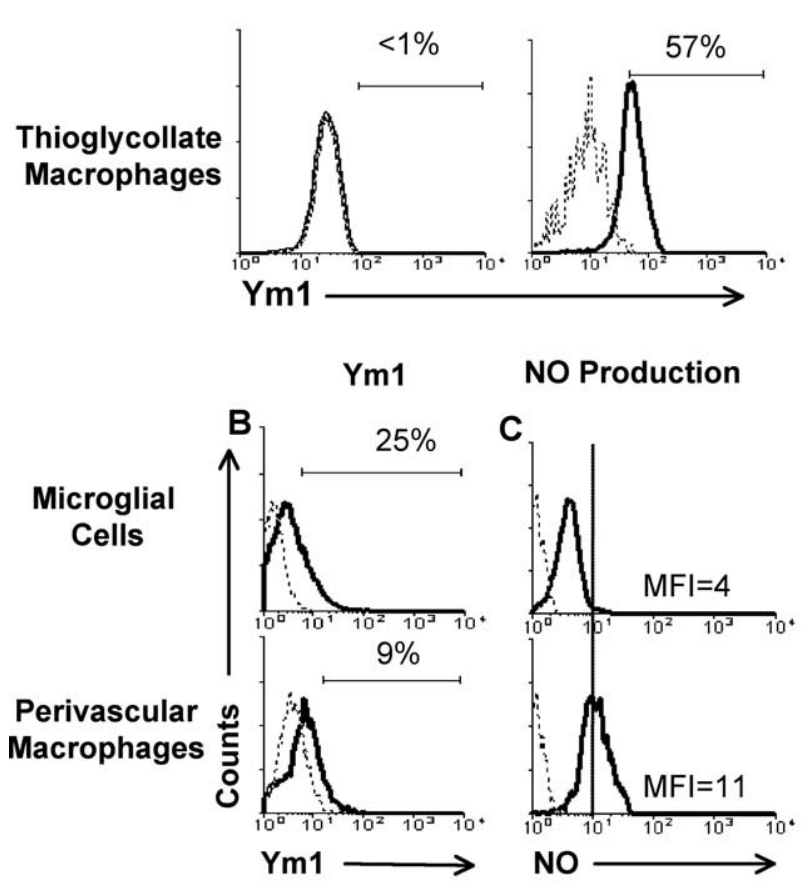

Microglial Cells

WVerivascular Macrophages
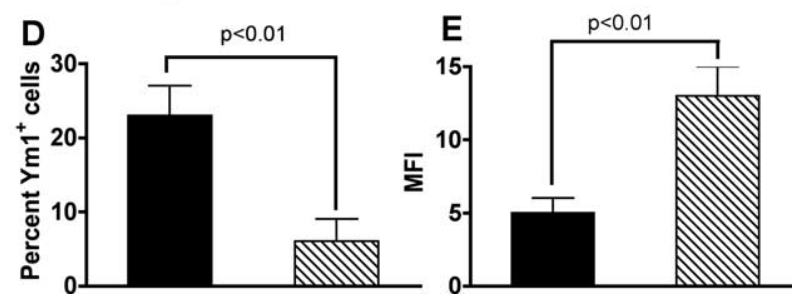

Figure 2. Comparison of Ym1 protein expression and N0 production by CNS microglial cells and perivascular macrophages. A, Peritoneal macrophages were stimulated with IL-4 (200 $\mathrm{U} / \mathrm{ml}$ ) or IFN- $\gamma(150 \mathrm{U} / \mathrm{ml})$ combined with LPS (100 $\mathrm{ng} / \mathrm{ml})$ for $24 \mathrm{~h}$ before staining for Ym1. $\boldsymbol{B}-\boldsymbol{E}$, Total mononuclear cells were isolated from the CNS of groups of four to five unmanipulated B10.PL mice, and microglial cells and perivascular macrophages were analyzed for Ym1 protein expression using intracellular staining $(\boldsymbol{B})$ and N0 production using the DAF-FM fluorescent probe $(\boldsymbol{C})$. $\boldsymbol{A}-\boldsymbol{C}$, The solid line represents staining with anti-Ym $1(\boldsymbol{A}, \boldsymbol{B})$ or DAF-FM probe fluorescence $(C$, and the dotted line represents background staining using rabbit serum $(A, B)$ or no probe ( $C$. $A, B$, The horizontal bar indicates positive staining, and the number above the bar is the percentage positive. $C$, The MFI for DAF-FM probe fluorescence is given. $A-C$, One representative of three experiments; $\boldsymbol{D}, \boldsymbol{E}$, mean $\pm S D$ of three separate experiments. $\boldsymbol{D}, \boldsymbol{E}$, There was a statistically significant ( $p \leq 0.01$ ) difference in the percentage of Ym1-positive cells $(\boldsymbol{D})$ and the level of N0 production $(\boldsymbol{E})$ in populations of microglial cells when compared with perivascular macrophages, as determined by the unpaired $t$ test.

average percentage of $\mathrm{Ym}^{+}$cells from three separate experiments is shown in Figure $3 B$.

In parallel to analysis of Ym 1 expression, we also analyzed NO production during EAE. Again, we used lymphocytes as the negative control. The solid line indicates the level of autofluorescence by the fluorescent probe in the lymphocytes. We found that the level of NO production by $\mathrm{CD} 45^{\text {low }}$ microglial cells was very low and did not exceed the background level of NO production by lymphocytes. Although the MFI of the CD $45^{\text {high }}$ microglial cells was slightly increased compared with CD $45^{\text {low }}$ cells, the level did not exceed the lymphocyte negative control (Fig. $3 A$ ). In contrast, production of NO by peripheral infiltrating macrophages was detected (Fig. $3 A$ ). As shown in Figure $4 C$, both CD $45^{\text {low }}$ and CD $45^{\text {high }}$ microglial

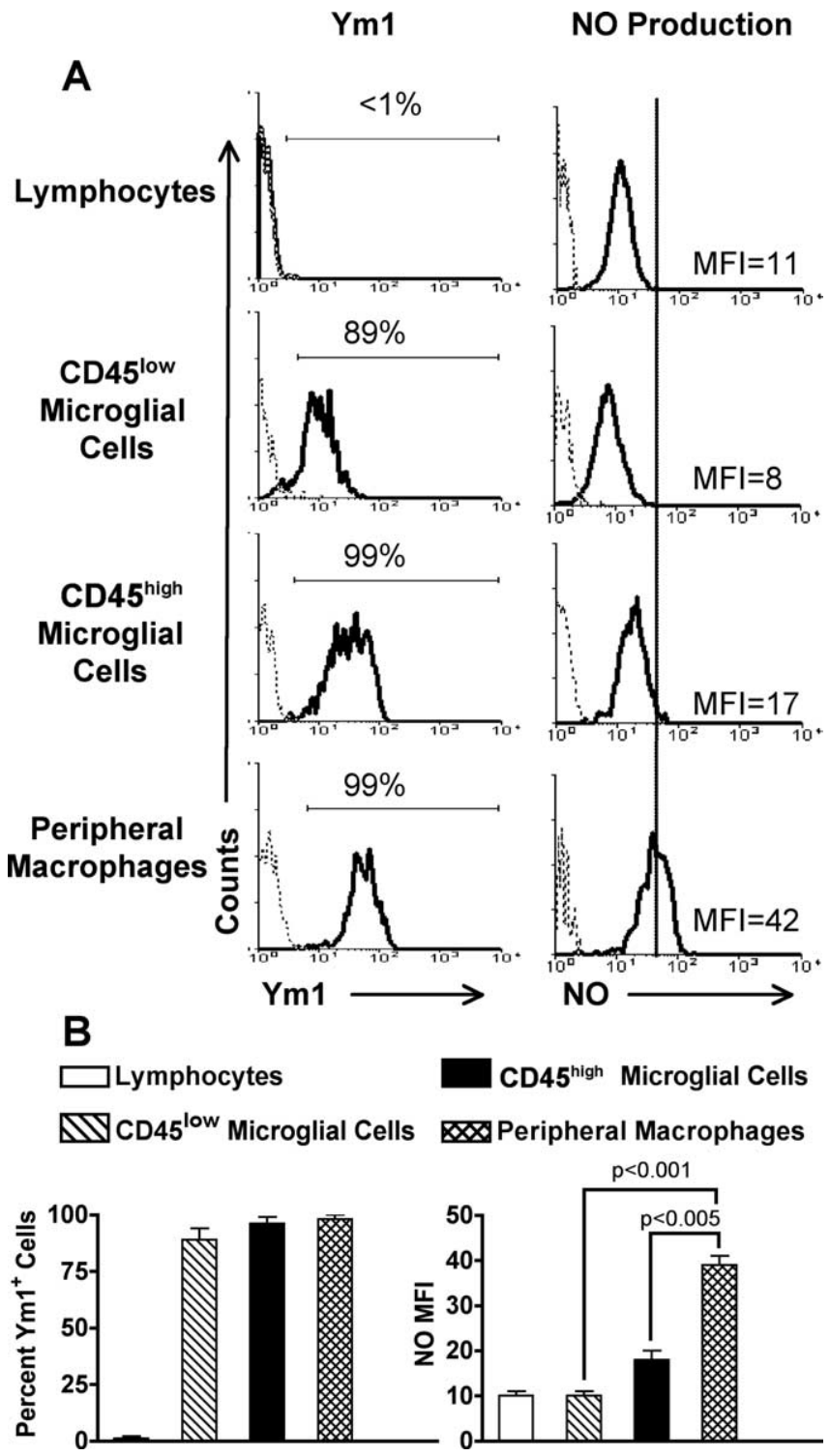

Figure 3. Ym1 protein expression and N0 production in CNS mononuclear cells from BM chimeric mice with EAE. Total mononuclear cells were isolated from the CNS of groups of four to five BM chimeras $10 \mathrm{~d}$ after EAE induction and stained for expression of surface markers as for Figure 1. Gated populations of mononuclear cells were assessed for Ym1 protein expression and N0 production as for Figure 2. $\boldsymbol{A}$, The solid line represents staining with anti-Ym1 or DAF-FM probe fluorescence, and the dotted line represents background staining using rabbit serum or no probe. The horizontal bar indicates positive staining, and the number above the bar is the percentage positive. The MFIs for DAF-FM fluorescence are shown. One representative experiment of three is shown. $\boldsymbol{B}$, Mean $\pm S D$ of three separate experiments. There was a statistically significant lower level of N0 production in both CD45 ${ }^{\text {low }}$ and CD $45^{\text {high }}$ microglial cells than in peripheral infiltrating macrophages, as determined by the unpaired $t$ test.

cells produced NO at a significantly lower level than peripheral infiltrating macrophages. The simultaneous expression of Yml and NO by macrophages suggests that the CNS microenvironment actively drives a new previously undescribed population of macrophages that exhibits characteristics of both aaM $\Phi$ s and caMФs. We have termed these macrophages dually activated macrophages (daM $\Phi$ s). Ym1 expression by microglial cells in the absence of NO demonstrates that the CNS microenvironment supports and maintains their aaM $\Phi$ phenotype, even during inflammation associated with EAE. 

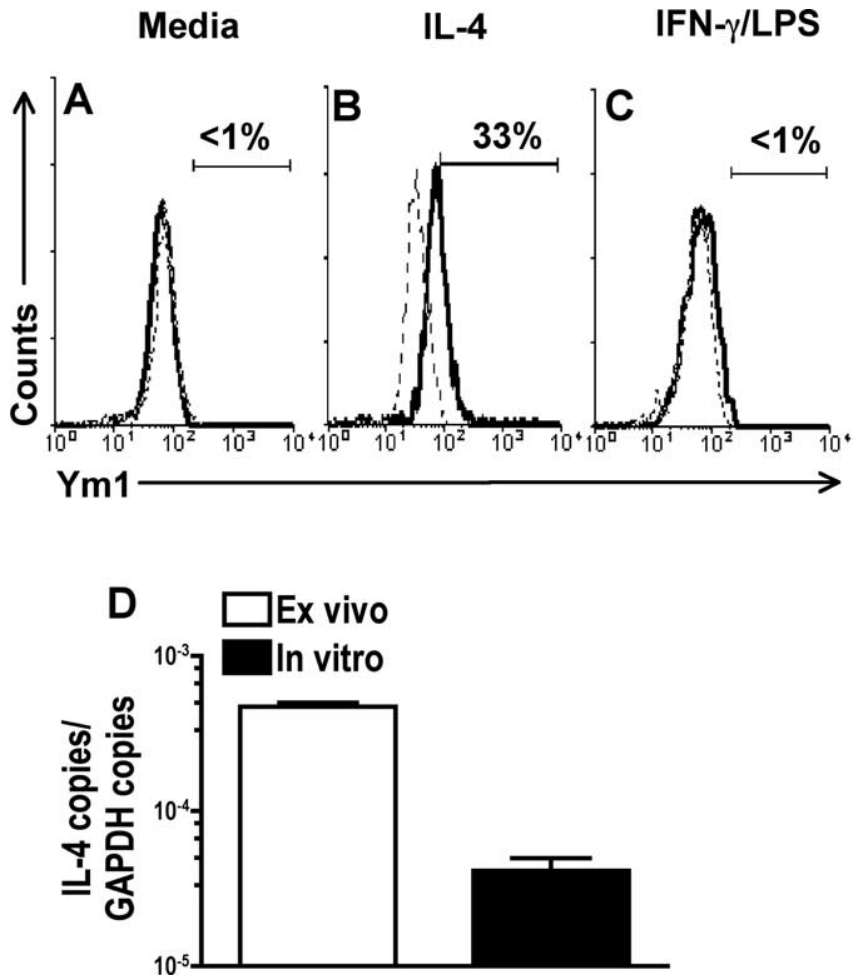

Figure 4. IL-4 induces the expression of Ym 1 in cultured microglial cells. $A-C$, Microglial cells cultured from adult mice were examined for expression of $\mathrm{Ym} 1$ by flow cytometry in medium alone $(\boldsymbol{A})$ or after incubation with IL-4 $(200 \mathrm{U} / \mathrm{ml})(\boldsymbol{B})$ or IFN- $\gamma(150 \mathrm{U} / \mathrm{ml})$ in combination with LPS (100 ng/ml) (C) for $24 \mathrm{~h}$. The solid line represents staining with anti-Ym1, and the dotted line represents background staining using rabbit serum (1:1000). The horizontal bar indicates positive staining, and the number above the bar is the percentage positive. $\boldsymbol{D}$, IL-4 mRNA levels were measured in ex vivo isolated (open bar) and in vitro cultured (filled bar) microglial cells by real-time RT-PCR as for Figure 1. $\boldsymbol{A}-\boldsymbol{D}, 0$ ne representative experiment of three is shown.

IL-4 induces Ym1 expression in cultured microglial cells

Ym1 expression by macrophages is known to be induced by IL-4. To confirm that IL-4 also upregulates Ym1 expression in microglial cells, we generated microglial cell cultures from adult B10.PL mice (Ponomarev et al., 2005a) and examined Ym1 expression by flow cytometry. Microglial cells cultured in medium alone did not express Ym1 protein (Fig. 4A), but the addition of IL-4 induced its expression (Fig. $4 B$ ). The addition of IFN- $\gamma$ in combination with LPS to induce classic activation did not result in Ym1 expression, as expected (Fig. 4C). To determine whether the lack of Ym 1 expression by in vitro cultured microglial cells was caused by reduced production of IL-4, we performed real-time PCR comparing IL-4 message levels with ex vivo isolated microglial cells. We found that cultured microglial cells expressed 10 -fold lower levels of IL-4 message than those isolated directly from the CNS (Fig. 4D). These cumulative data confirm that IL-4 induces Ym1 expression in microglial cells and suggest that the CNS microenvironment signals IL-4 production by microglial cells.

IL-4 production in the CNS is required for the maintenance of Yml expression and suppression of EAE

We found that microglial cells expressed mRNA for IL-4 in vivo, suggesting that they are the major source of IL- 4 in normal CNS. To investigate whether IL-4 and/or IL-13 were required for maintenance of Ym1 expression in microglial cells, we used mice deficient in IL-4 production (IL-4 ${ }^{-/-}$) and mice deficient in the IL- $4 \alpha \mathrm{R}$, which is common to both the IL- 4 and IL-13 receptors (Hilton et al., 1996). Because IL- $4^{-1-}$ mice were on the C57BL/6

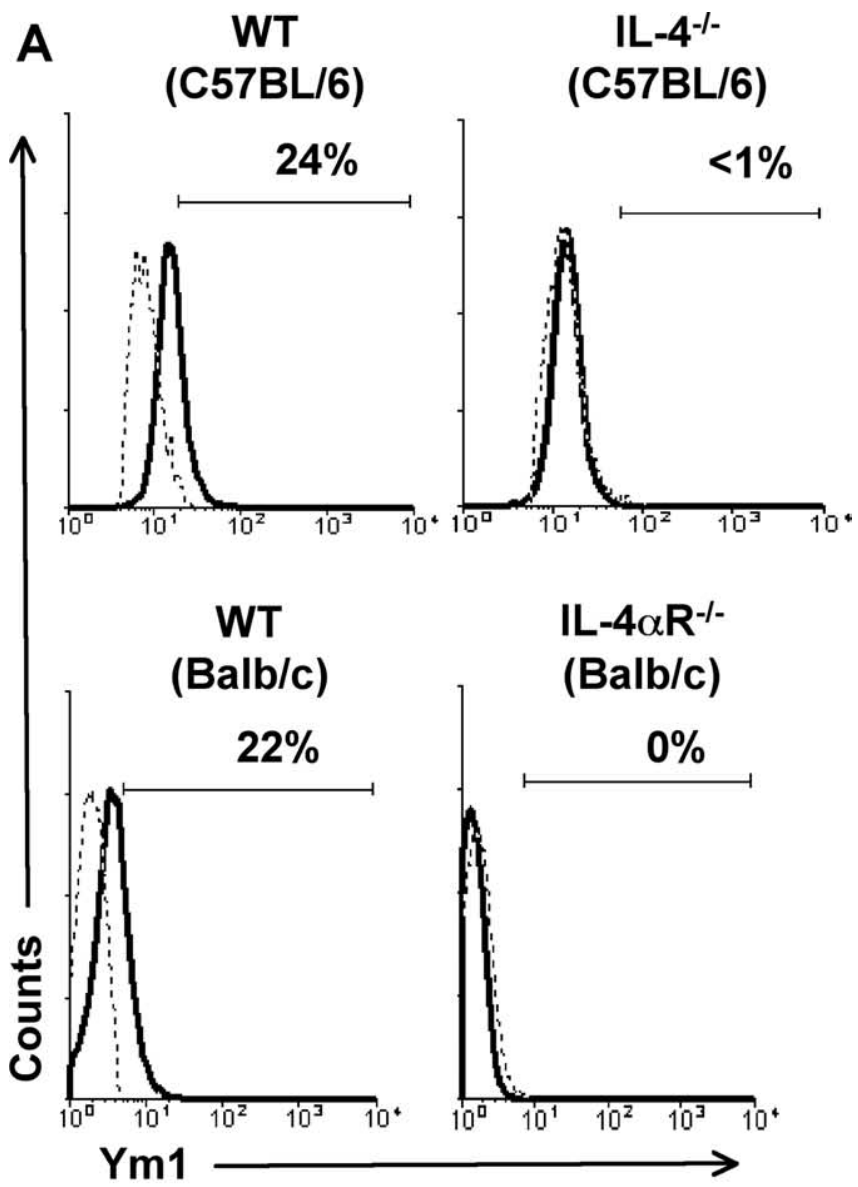

B

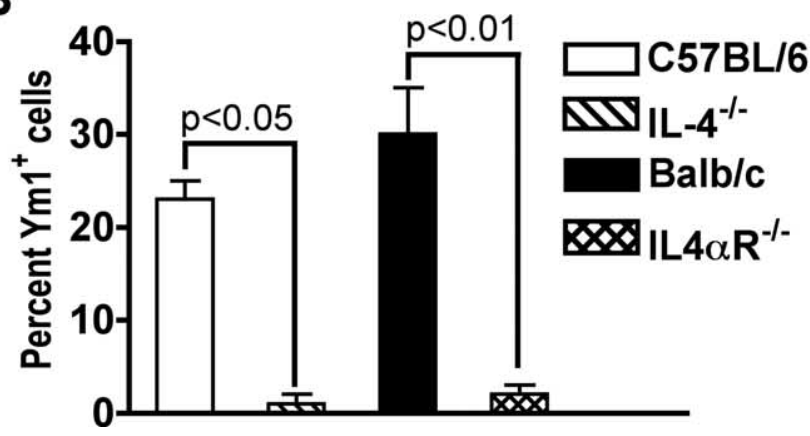

Figure 5. Ym1 protein expression in microglial cells is IL-4 dependent. Total mononuclear cells were isolated from the CNS of groups of four to five C57BL/6, IL-4 $4^{-1-}, \mathrm{BALB} / \mathrm{C}$, and IL- $4 \alpha \mathrm{R}^{-1-}$ mice and stained for expression of surface markers and $\mathrm{Ym} 1$. $A$, The solid line represents staining with anti-Ym1, and the dotted line represents background staining using rabbit serum. The horizontal bar indicates positive staining, and the number above the bar is the percentage positive. One representative experiment of three is shown. $\boldsymbol{B}$, Mean \pm SD of three separate experiments. There was a statistically significant decrease in the percentage of $\mathrm{Ym}{ }^{+}$ microglial cells in both IL-4- and IL-4 $\alpha$ R-deficient mice when compared with wild-type control animals, as determined by the unpaired $t$ test.

background and IL- $4 \alpha \mathrm{R}^{-/-}$mice were on the BALB/c background, we first investigated whether microglial cells from these two genetic backgrounds expressed comparable levels of Ym1 protein. We found that $20-30 \%$ of microglial cells from both mice expressed Ym1 (Fig. 5A). When we investigated the level of $\mathrm{Ym} 1$ protein in microglial cells in IL- $4^{-1-}$ and IL- $4 \alpha \mathrm{R}^{-/-}$mice, we found that microglial cells from both mice were negative for Ym1 expression (Fig. 5A). An average of three experiments is shown in Figure $5 B$. These data indicate that IL- 4 alone is suffi- 
Table 1. Summary of disease course and cell parameters in WT $\rightarrow$ WT and WT $\rightarrow$ IL$4^{-/-}$BM chimera mice ${ }^{a}$

\begin{tabular}{|c|c|c|}
\hline & $\mathrm{WT} \rightarrow \mathrm{WT}$ & $\mathrm{WT} \rightarrow \mathrm{IL}-4^{-1-}$ \\
\hline Day of onset ${ }^{b}$ & $14.3 \pm 0.7$ & $11.6 \pm 0.5^{c}$ \\
\hline Day 18 average score & $1.9 \pm 0.3$ & $2.9 \pm 0.1^{d}$ \\
\hline Number of mice & 9 & 13 \\
\hline Total number of mononuclear cells $\left(\times 10^{3}\right)$ & $310 \pm 21$ & $640 \pm 90^{e}$ \\
\hline Absolute number of lymphocytes $\left(\times 10^{3}\right)^{f}$ & $109 \pm 9$ & $274 \pm 26^{c}$ \\
\hline $\begin{array}{l}\text { Absolute number of resting } C D 45^{\text {low }} \text { microglial cells } \\
\quad\left(\times 10^{3}\right)^{f}\end{array}$ & $115 \pm 17$ & $143 \pm 22$ \\
\hline $\begin{array}{l}\left(\times 10^{3}\right)^{f} \\
\text { Absolute number of peripheral macrophages }\left(\times 10^{3}\right)^{f}\end{array}$ & $\begin{array}{l}47 \pm 11 \\
37 \pm 4\end{array}$ & $\begin{array}{c}127 \pm 19^{e} \\
74 \pm 7^{e}\end{array}$ \\
\hline
\end{tabular}

a Lethally irradiated WT or IL-4 ${ }^{-1-}$ C57BL/6 mice were transplanted with BM from (C57BL/6xB10.PL)F $\mathrm{F}_{1}$ mice and allowed to reconstitute for 8 weeks, and EAE was induced by immunization with $M \mathrm{GG}_{35-55}$ peptide. The data shown are the average $\pm S E$ of three experiments with three to five mice per group observed on day 18 after EAE induction.

${ }^{b}$ Graded disease score as described in Materials and Methods.

${ }^{c} p<0.005$ when compared to WT $\rightarrow$ WT.

${ }^{d} p<0.01$ when compared to WT $\rightarrow$ WT.

$e_{p}<0.05$ when compared to WT $\rightarrow$ WT.

${ }^{f}$ Absolute numbers were determined by multiplying the total cell count obtained by counting on a hemocytometer by the percentage of lymphocytes, resting and activated microglial cells, and peripheral macrophages determined by flow cytometry as for Figure 3 and then dividing by the number of mice in each group.

cient for the induction of Ym1 expression in microglial cells in the CNS.

To assess whether IL-4 expression by CNS-resident cells is important for regulation of EAE disease and maintenance of the $\mathrm{Ym}^{+}{ }^{+}$aaM $\Phi$ phenotype, we generated chimera mice with a deficiency in IL-4 in the CNS by transplanting BM from wild-type (WT) (B10.PLxC57BL/6) $\mathrm{F}_{1}$ mice into lethally irradiated IL-4 ${ }^{-1-}$ $\mathrm{C} 57 \mathrm{BL} / 6$ animals $\left(\mathrm{WT} \rightarrow \mathrm{IL}-4^{-1-}\right)$. When we induced $\mathrm{EAE}$ in the chimeras, we found that WT $\rightarrow$ IL-4 chimeras had exacerbated EAE with an earlier onset, compared with control chimeras (Fig. $5 A$, Table 1). The average day of onset for the $\mathrm{WT} \rightarrow \mathrm{WT}$ chimeras was $14.3 \pm 0.7$, compared with $11.6 \pm 0.5$ in the $\mathrm{WT} \rightarrow \mathrm{IL}_{-} 4^{-1-}$ mice. This difference was statistically significant $(p<0.005)$. On day 18 after disease induction, the average daily score was $2.9 \pm$ 0.1 in the $\mathrm{WT} \rightarrow \mathrm{IL}-4^{-1-}$ and $1.9 \pm 0.3$ in the WT $\rightarrow$ WT chimera mice, which was a significant increase $(p<0.01)$ (Fig. 6A, Table $1)$. Because of the severe illness of the WT $\rightarrow \mathrm{IL}-4^{-1-}$ chimera mice, the experiment was terminated on day 18 , and mononuclear cells from the CNS were isolated for analysis of leukocyte infiltration and $\mathrm{Ym} 1$ expression.

$\mathrm{WT} \rightarrow \mathrm{IL}-4^{-1-}$ chimeras had statistically significant twofold increase in the absolute number of infiltrating lymphocytes and peripheral infiltrating macrophages (Table 1). Similarly, the absolute number of activated microglial cells was also statistically increased in the WT $\rightarrow$ IL-4 $4^{-1-}$ chimeras, whereas the slight increase in resting microglial cells did not reach significance (Table 1). Ym1 expression by CD $45^{\text {low }}$ and CD $45^{\text {high }}$ microglial cells and peripheral infiltrating macrophages was essentially absent in the $\mathrm{WT} \rightarrow \mathrm{IL}-4^{-1-}$ chimeras compared with $\mathrm{WT} \rightarrow \mathrm{WT}$ control (Fig. $6 \mathrm{~B}$ ). Thus IL-4 expression by CNS-resident cells is essential for the maintenance of an aaM $\Phi$ phenotype by both microglial cells and peripheral infiltrating macrophages in the CNS and for the suppression of EAE clinical symptoms.

\section{Discussion}

In this study, we investigated the function of IL-4 in the CNS and found that its expression within the CNS was required for the regulation of EAE by modulating the extent of the inflammatory infiltrate. In the absence of CNS-derived IL-4, the absolute number of both lymphocytes and peripheral infiltrating macrophages
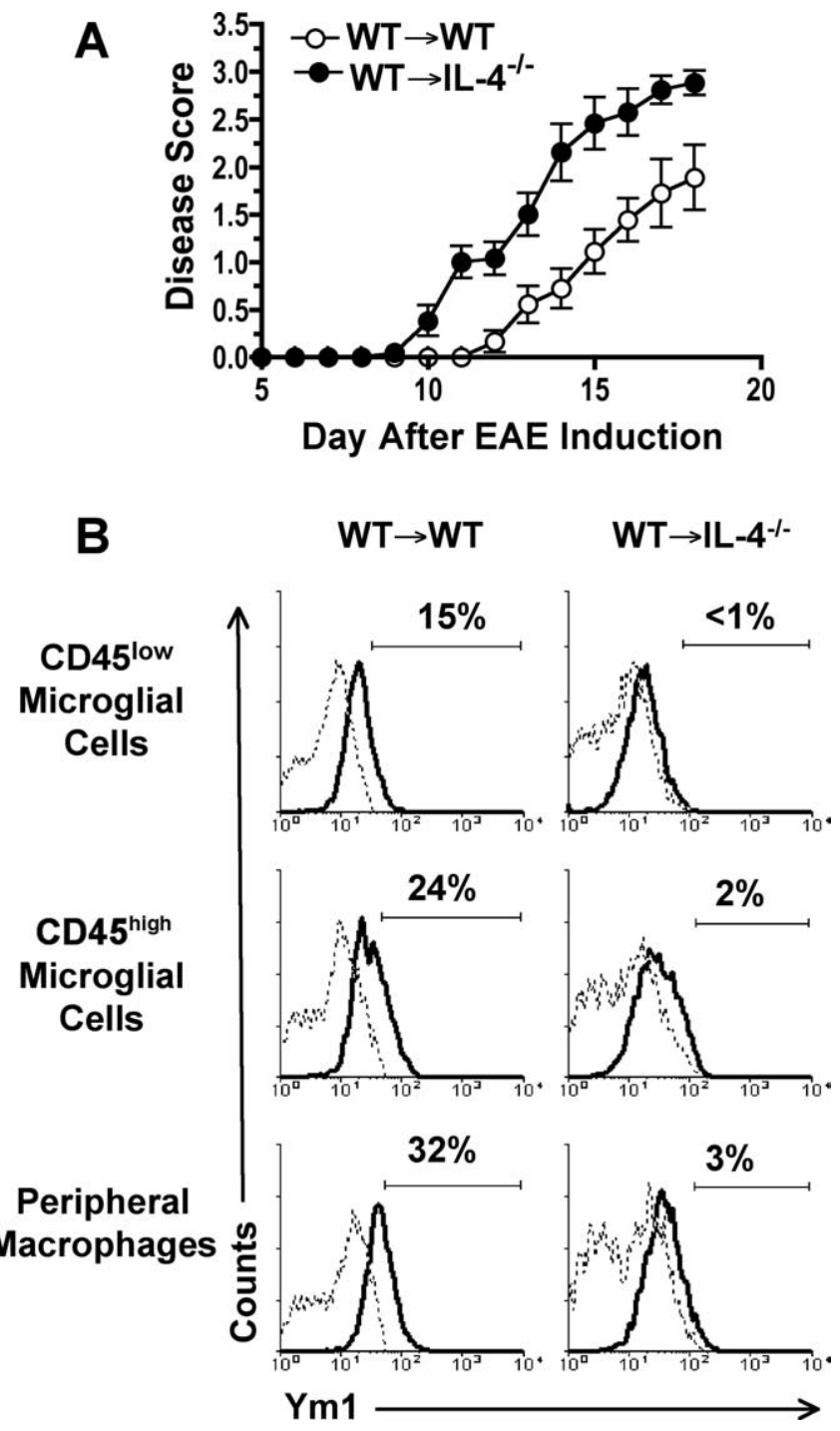

Figure 6. EAE is more severe in mice with a CNS deficiency in IL-4. Lethally irradiated WT $(\mathrm{WT} \rightarrow \mathrm{WT})$ or IL-4 ${ }^{-1-}\left(\mathrm{WT} \rightarrow \mathrm{IL}-4^{-1-}\right)$ C57BL/6 mice were transplanted with BM from $(\mathrm{C} 57 \mathrm{BL} / 6 \mathrm{xB10} . \mathrm{PL}) \mathrm{F}_{1}$ mice and allowed to reconstitute for 8 weeks before induction of EAE by immunization with $\mathrm{MOG}_{35-55}$ peptide. $A$, Mean $\pm S D$ of the daily scores of individual mice combined from three separate experiments with three to five mice in each group. $\boldsymbol{B}$, The same groups of animals shown in $\boldsymbol{A}$ were used for isolation of mononuclear cells from the CNS on day 18 after EAE induction and flow cytometric analysis of Ym1 expression in microglial cells and peripheral infiltrating macrophages. Gated population of CD45 ${ }^{\text {low }}$ resting microglial cells, CD45 high microglial cells, and peripheral infiltrating macrophages were analyzed for Ym1 expression. The solid line represents staining with anti-Ym1, and the dotted line represents background staining using rabbit serum. The horizontal bar indicates positive staining, and the number above the bar is the percentage positive. The data shown are representative of three separate experiments.

were significantly increased, resulting in more severe EAE. The CNS-derived IL-4 also influenced the phenotype of both microglial cells and macrophages in the CNS by inducing the expression of Ym1, marking them as alternatively activated. Thus, IL-4 production within the CNS is critical for the regulation of CNS inflammation.

The cellular source of the CNS-derived IL-4 could be a number of cell types. We show that one of those sources is microglial cells themselves (Fig. 1). Thus, they have the capacity to autoregulate themselves. Another potential cellular source is astrocytes (Hulshof et al., 2002). Because IL-4 production in the normal CNS is not detectable, astrocytes would likely only produce 
IL-4 after activation during the EAE inflammatory process. In our model, during EAE, the encephalitogenic T-cells are not likely to contribute substantial amounts of IL-4. This is because of their Th1 phenotype (Dittel et al., 1999) and because EAE was more severe in the BM chimeras devoid of IL- 4 in the CNS (Fig. $5)$. Mice in which IL-4 was expressed in T- and B-cells were not protected from EAE, suggesting that these endogenous cells are also not a significant source of IL-4 (Tepper et al., 1990). Another potential source of IL- 4 is mast cells, but they have been shown to exert their effects outside of the CNS (Tanzola et al., 2003). Thus, in the CNS, the primary source of IL-4 is likely microglial cells.

The signals that drive IL-4 production by microglial cells in the CNS are not known. Our data showing that ex vivo isolated microglial cells produce 10 -fold more IL-4 message than cultured microglial cells suggest that the CNS microenvironment influences microglial cell function. Because microglial cells are distributed throughout the entire CNS, they have the potential to be in contact with all of the CNS cellular components. Functional interactions between microglial cells and astrocytes, oligodendrocytes, and neurons have been described (Dobrenis et al., 2005; Filipovic and Zecevic, 2005; Satoh et al., 2005; Mohri et al., 2006; Hasegawa et al., 2007). Because IL-4 message increased in microglial cells during EAE inflammation, an interesting speculation is that this production is caused by microglial cells responding to inflammatory signals from either oligodendrocytes undergoing cell death or activated astrocytes. The consequence would be the increased production of an anti-inflammatory cytokine with the potential to regulate the proinflammatory activity of astrocytes (Molina-Holgado et al., 2002).

Our data showing that IL-4 induces the expression of the Ym1 protein by both microglial cells and macrophages in the CNS suggest that the aaM $\Phi$ phenotype is important for regulation of inflammation associated with EAE. An increase in Ym1 mRNA expression was recently shown in one of two analyzed mouse models of Alzheimer's disease (Colton et al., 2006). Although the exact function of microglial cells is still being debated in neurodegenerative diseases, it was recently shown that IL-4-treated microglial cells injected into the CSF of mice with EAE suppressed clinical symptoms and promoted oligodendrogenesis in the spinal cord (Butovsky et al., 2006). In addition, the addition of IL-4 to LPS-stimulated microglial and motoneuron cocultures suppressed the release of $\mathrm{NO}$ and decreased motoneuron injury (Zhao et al., 2006). These studies suggest that IL-4-induced microglial cells are both anti-inflammatory and neuroprotective. Our studies demonstrating that IL-4 production within the CNS induces aaM $\Phi$ s and regulates the extent of inflammation support this conclusion.

We confirmed that infiltrating peripheral macrophages produce NO in the CNS. The role of NO in regulating EAE is not straightforward, with inhibitors of iNOS showing both protection and exacerbation in a variety of EAE models (Bogdan, 1998). Mice deficient in iNOS have exacerbated disease (Fenyk-Melody et al., 1998; Sahrbacher et al., 1998). What is becoming clear is that NO has dual functions in the CNS and that the timing and site of NO production determines its function (Bogdan, 1998; O'Brien et al., 2001). Interestingly, we did not detect NO production by activated microglial cells during EAE (Fig. 3). This finding, along with their Ym1 expression, categorizes microglial cells as aaMФs. Also of interest is the coexpression of $\mathrm{NO}, \mathrm{Ym} 1$, and FIZZ1 in the peripheral infiltrating macrophage population, suggesting that these daM $\Phi$ s may also play a role in downmodulating CNS inflammation.

The CNS is a unique tissue with the presence of specialized glial cells important for neuronal function. Maintaining an immunosuppressive microenvironment in the CNS is important to prevent irreversible tissue damage. The inefficient induction of immune responses in the CNS is evident by the inability of the immune system to clear viruses that are harbored within the CNS. The exact mechanisms the CNS uses to maintain its immunosuppressive state are not clearly understood. Our data demonstrate that the production of IL-4 in the CNS is one such mechanism, providing additional evidence that IL-4 is a potential therapeutic target in the treatment of CNS inflammatory disorders that include MS.

\section{References}

Bettelli E, Das MP, Howard ED, Weiner HL, Sobel RA, Kuchroo VK (1998) IL-10 is critical in the regulation of autoimmune encephalomyelitis as demonstrated by studies of IL-10- and IL-4-deficient and transgenic mice. J Immunol 161:3299-3306.

Bogdan C (1998) The multiplex function of nitric oxide in (auto)immunity. J Exp Med 187:1361-1365.

Butovsky O, Landa G, Kunis G, Ziv Y, Avidan H, Greenberg N, Schwartz A, Smirnov I, Pollack A, Jung S, Schwartz M (2006) Induction and blockage of oligodendrogenesis by differently activated microglia in an animal model of multiple sclerosis. J Clin Invest 116:905-915.

Carson MJ (2002) Microglia as liaisons between the immune and central nervous systems: functional implications for multiple sclerosis. Glia 40:218-231.

Chang NC, Hung SI, Hwa KY, Kato I, Chen JE, Liu CH, Chang AC (2001) A macrophage protein, Ym1, transiently expressed during inflammation is a novel mammalian lectin. J Biol Chem 276:17497-17506.

Colton CA, Mott RT, Sharpe H, Xu Q, Van Nostrand WE, Vitek MP (2006) Expression profiles for macrophage alternative activation genes in $\mathrm{AD}$ and in mouse models of AD. J Neuroinflammation 3:27.

Dittel BN, Merchant RM, Janeway Jr CA (1999) Evidence for Fasdependent and Fas-independent mechanisms in the pathogenesis of experimental autoimmune encephalomyelitis. J Immunol 162:6392-6400.

Dobrenis K, Chang HY, Pina-Benabou MH, Woodroffe A, Lee SC, Rozental R, Spray DC, Scemes E (2005) Human and mouse microglia express connexin36, and functional gap junctions are formed between rodent microglia and neurons. J Neurosci Res 82:306-315.

Edwards JP, Zhang X, Frauwirth KA, Mosser DM (2006) Biochemical and functional characterization of three activated macrophage populations. J Leukoc Biol 80:1298-1307.

Falcone M, Rajan AJ, Bloom BR, Brosnan CF (1998) A critical role for IL-4 in regulating disease severity in experimental allergic encephalomyelitis as demonstrated in IL-4-deficient C57BL/6 mice and BALB/c mice. J Immunol 160:4822-4830.

Fenyk-Melody JE, Garrison AE, Brunnert SR, Weidner JR, Shen F, Shelton BA, Mudgett JS (1998) Experimental autoimmune encephalomyelitis is exacerbated in mice lacking the NOS2 gene. J Immunol 160:2940-2946.

Filipovic R, Zecevic N (2005) Interaction between microglia and oligodendrocyte cell progenitors involves Golli proteins. Ann NY Acad Sci 1048:166-174.

Furlan R, Poliani PL, Galbiati F, Bergami A, Grimaldi LM, Comi G, Adorini L, Martino G (1998) Central nervous system delivery of interleukin 4 by a nonreplicative herpes simplex type 1 viral vector ameliorates autoimmune demyelination. Hum Gene Ther 9:2605-2617.

Hasegawa S, Yamaguchi M, Nagao H, Mishina M, Mori K (2007) Enhanced cell-to-cell contacts between activated microglia and pyramidal cell dendrites following kainic acid-induced neurotoxicity in the hippocampus. J Neuroimmunol 186:75-85.

Hilton DJ, Zhang JG, Metcalf D, Alexander WS, Nicola NA, Willson TA (1996) Cloning and characterization of a binding subunit of the interleukin 13 receptor that is also a component of the interleukin 4 receptor. Proc Natl Acad Sci USA 93:497-501.

Hulshof S, Montagne L, De Groot CJ, Van Der Valk P (2002) Cellular localization and expression patterns of interleukin-10, interleukin-4, and their receptors in multiple sclerosis lesions. Glia 38:24-35.

Jin HM, Copeland NG, Gilbert DJ, Jenkins NA, Kirkpatrick RB, Rosenberg M (1998) Genetic characterization of the murine Yml gene and identification of a cluster of highly homologous genes. Genomics 54:316-322.

Mohri I, Taniike M, Taniguchi H, Kanekiyo T, Aritake K, Inui T, Fukumoto 
N, Eguchi N, Kushi A, Sasai H, Kanaoka Y, Ozono K, Narumiya S, Suzuki K, Urade Y (2006) Prostaglandin D2-mediated microglia/astrocyte interaction enhances astrogliosis and demyelination in twitcher. J Neurosci 26:4383-4393.

Molina-Holgado E, Arevalo-Martin A, Castrillo A, Bosca L, Vela JM, Guaza C (2002) Interleukin-4 and interleukin-10 modulate nuclear factor kappaB activity and nitric oxide synthase-2 expression in Theiler's virus-infected brain astrocytes. J Neurochem 81:1242-1252.

Nelson PT, Soma LA, Lavi E (2002) Microglia in diseases of the central nervous system. Ann Med 34:491-500.

Nimmerjahn A, Kirchhoff F, Helmchen F (2005) Resting microglial cells are highly dynamic surveillants of brain parenchyma in vivo. Science 308:1314-1318.

Nio J, Fujimoto W, Konno A, Kon Y, Owhashi M, Iwanaga T (2004) Cellular expression of murine $\mathrm{Ym} 1$ and $\mathrm{Ym} 2$, chitinase family proteins, as revealed by in situ hybridization and immunohistochemistry. Histochem Cell Biol 121:473-482.

O'Brien NC, Charlton B, Cowden WB, Willenborg DO (2001) Inhibition of nitric oxide synthase initiates relapsing remitting experimental autoimmune encephalomyelitis in rats, yet nitric oxide appears to be essential for clinical expression of disease. J Immunol 167:5904-5912.

Okuda Y, Nakatsuji Y, Fujimura H, Esumi H, Ogura T, Yanagihara T, Sakoda $S$ (1995) Expression of the inducible isoform of nitric oxide synthase in the central nervous system of mice correlates with the severity of actively induced experimental allergic encephalomyelitis. J Neuroimmunol 62:103-112.

Overbergh L, Valckx D, Waer M, Mathieu C (1999) Quantification of murine cytokine mRNAs using real time quantitative reverse transcriptase PCR. Cytokine 11:305-312.

Ponomarev ED, Dittel BN (2005) $\gamma \delta$ T cells regulate the extent and duration of inflammation in the central nervous system by a Fas ligand-dependent mechanism. J Immunol 174:4678-4687.

Ponomarev ED, Novikova M, Yassai M, Szczepanik M, Gorski J, Dittel BN (2004) $\gamma \delta$ T cell regulation of IFN- $\gamma$ production by central nervous system-infiltrating encephalitogenic $\mathrm{T}$ cells: correlation with recovery from experimental autoimmune encephalomyelitis. J Immunol 173:1587-1595.

Ponomarev ED, Novikova M, Maresz K, Shriver LP, Dittel BN (2005a) Development of a culture system that supports adult microglial cell proliferation and maintenance in the resting state. J Immunol Methods 300:32-46.

Ponomarev ED, Shriver LP, Maresz K, Dittel BN (2005b) Microglial cell activation and proliferation precedes the onset of CNS autoimmunity. J Neurosci Res 81:374-389.
Raes G, De Baetselier P, Noel W, Beschin A, Brombacher F, Hassanzadeh Gh G (2002) Differential expression of FIZZ1 and Ym1 in alternatively versus classically activated macrophages. J Leukoc Biol 71:597-602.

Sahrbacher UC, Lechner F, Eugster HP, Frei K, Lassmann H, Fontana A (1998) Mice with an inactivation of the inducible nitric oxide synthase gene are susceptible to experimental autoimmune encephalomyelitis. Eur J Immunol 28:1332-1338.

Satoh J, Onoue H, Arima K, Yamamura T (2005) Nogo-A and nogo receptor expression in demyelinating lesions of multiple sclerosis. J Neuropathol Exp Neurol 64:129-138.

Sedgwick JD, Schwender S, Imrich H, Dorries R, Butcher GW, ter Meulen V (1991) Isolation and direct characterization of resident microglial cells from the normal and inflamed central nervous system. Proc Natl Acad Sci USA 88:7438-7442.

Shaw MK, Lorens JB, Dhawan A, DalCanto R, Tse HY, Tran AB, Bonpane C, Eswaran SL, Brocke S, Sarvetnick N, Steinman L, Nolan GP, Fathman CG (1997) Local delivery of interleukin 4 by retrovirus-transduced T lymphocytes ameliorates experimental autoimmune encephalomyelitis. J Exp Med 185:1711-1714.

Stein M, Keshav S, Harris N, Gordon S (1992) Interleukin 4 potently enhances murine macrophage mannose receptor activity: a marker of alternative immunologic macrophage activation. J Exp Med 176:287-292.

Tanzola MB, Robbie-Ryan M, Gutekunst CA, Brown MA (2003) Mast cells exert effects outside the central nervous system to influence experimental allergic encephalomyelitis disease course. J Immunol 171:4385-4391.

Tepper RI, Levinson DA, Stanger BZ, Campos-Torres J, Abbas AK, Leder P (1990) IL-4 induces allergic-like inflammatory disease and alters T cell development in transgenic mice. Cell 62:457-467.

Tran EH, Hardin-Pouzet H, Verge G, Owens T (1997) Astrocytes and microglia express inducible nitric oxide synthase in mice with experimental allergic encephalomyelitis. J Neuroimmunol 74:121-129.

Webb DC, McKenzie AN, Foster PS (2001) Expression of the Ym2 lectinbinding protein is dependent on interleukin (IL)- 4 and IL-13 signal transduction: identification of a novel allergy-associated protein. J Biol Chem 276:41969-41976.

Welch JS, Escoubet-Lozach L, Sykes DB, Liddiard K, Greaves DR, Glass CK (2002) TH2 cytokines and allergic challenge induce Ym1 expression in macrophages by a STAT6-dependent mechanism. J Biol Chem 277:42821-42829.

Zhao W, Xie W, Xiao Q, Beers DR, Appel SH (2006) Protective effects of an anti-inflammatory cytokine, interleukin- 4 , on motoneuron toxicity induced by activated microglia. J Neurochem 99:1176-1187. 